\title{
Irritation cutanée et vitesse de propagation d'ondes ultrasonores
}

\author{
E. Uhoda and G. E. Piérard \\ Service de Dermatopathologie, CHU du Sart Tilman, B-4000 Liège, Belgique
}

Received 18 December 2002, Accepted 3 February 2003

Keywords: irritation, stratum corneum, surfactant, ultrasound, xerosis

Mots-clés: irritation, couche cornée, tensio-actif, ultrason, xérose

\section{Synopsis}

Cutaneous irritation is a stereotyped response of the skin to any physical or chemical insult. Its main manifestations encompass inflammatory erythema and an alteration of the cutaneous microrelief showing increased roughness, dehydration and erratic desquamation. Several instrumental methods can indirectly assess the issues of cutaneous irritation on human stratum corneum.

The aim of the present study was to measure the speed of propagation of ultrasound shear waves in the skin irritated by anionic surfactants. The study was performed in 25 healthy subjects of both sexes. Occlusive patches of a $10 \%$ solution of anionic surfactants were applied to a forearm of each volunteer. The other forearm served as an untreated control site. Patches remained in place for $24 \mathrm{~h}$. Three hours later, another patch was placed again for $21 \mathrm{~h}$. Resonance running time measurements (RRTM) of the ultrasound shear wave were performed using a Reviscometer ${ }^{\circledR}$ RVM 600. Measurements were performed at entry in the study and 2 days after removing the second patch.

Desquamative xerosis was obvious at the sites patched with the surfactant solution. RRTM values were significantly $(P<0.0001)$ lower than those at the control site. Hence, the speed of propagation of ultrasound shear waves was shown to be increased at an irritated site compared to an intact site.

Correspondence: Gerald E. Piérard, Service de Dermatopathologie, CHU du Sart Tilman, B-4000 Liège, Belgique. E-mail: Gerald.Pierard@ulg.ac.be
In conclusion, this study shows that the propagation speed of ultrasounds is influenced by the skin surface nature, being considerably increased in skin altered by surfactants.

\section{Résumé}

L'irritation cutanée, réponse stéréotypée de la peau à toute agression physique ou chimique, a comme principales manifestations un érythème inflammatoire et une altération du relief cutané se marquant par une rugosité accrue, une déshydratation et une desquamation anarchique. Différentes méthodes instrumentales de mesure sont capables d'évaluer de façon indirecte les conséquences d'une irritation cutanée sur le stratum corneum.

Le but de cette étude était d'évaluer la vitesse de propagation d'ondes ultrasonores à travers une peau irritée par des tensio-actifs anioniques. L'étude a été réalisée chez 25 sujets sains des deux sexes. Des applications sous occlusion d'une solution à 10\% de tensio-actifs anioniques ont été appliquées sur un avant-bras de chaque volontaire. L'autre avant-bras a servi de site témoin non traité. Le contact a été maintenu avec la peau pendant $24 \mathrm{~h}$. Trois heures après, une autre application identique a été gardée sous occlusion pendant 21 heures. La mesure du temps de propagation d'ondes ultrasonores (RRTM, resonance running time measurement) a été effectuée à l'aide d'un Reviscometer ${ }^{\circledR}$ RVM 600. Cette évaluation a eu lieu préalablement à la mise en place des deux tests occlusifs et deux jours après l'enlèvement du second.

Une xérose desquamative s'est manifestée aux sites ayant été en contact avec la solution de surfactants. 
Les valeurs de RRTM y étaient significativement $(P<0.0001)$ inférieures à celles obtenues sur les sites non irrités. Dès lors, la vitesse de propagation dondes ultrasonores augmente sur un site irrité par rapport à un site sain.

En conclusion, létude démontre que la vitesse de propagation d'ondes ultrasonores est influencée par l'état de surface cutanée. Elle est nettement accélérée sur la peau altérée par des tensio-actifs.

\section{Introduction}

L'irritation cutanée représente une réponse stéréotypée de la peau à une agression chimique ou physique. Cette réponse inflammatoire est immédiate, locale et non immunologique. En particulier, les tensio-actifs interagissent avec la peau et provoquent une irritation cutanée [1,2].

Le stratum corneum, partie la plus superficielle de la peau, est le premier tissu biologique en contact avec toute agression extérieure; son rôle est très important dans la protection contre les effets de xénobiotiques irritants. Il est donc à la fois le témoin et la victime du processus d'irritation et correspond au niveau le plus accessible pour toute mesure instrumentale de ses manifestations cliniques et subcliniques. L'irritation cutanée se manifeste par un érythème inflammatoire et une altération du microrelief cutané se marquant par une rugosité accrue, une déshydratation superficielle, une desquamation anarchique et une perméabilité cutanée augmentée [3].

Différents instruments de mesure sont capables d'évaluer de façon indirecte les conséquences de l'irritation au niveau cutané. Parmi les plus classiques visant les fonctions de la couche cornée, l'Evaporimeter EP1 mesure la perte insensible en eau, alors que le Corneometer CM 820 et le NOVA Dermal Phase Meter 9003 évaluent par des mesures électriques l'état d'hydratation du stratum corneum [4-7].

La vitesse de propagation d'un train d'ondes ultrasonores dans la peau varie en fonction de certaines caractéristiques de ses tissus constitutifs. La plupart des travaux publiés à ce jour ont démontré l'influence des qualités physiques du derme [8-13]. La chevelure propage également les ondes ultrasonores à une vitesse variable selon lagencement des cheveux et leur structure plus ou moins altérée [14]. Aucune étude n’a été rapportée visant à explorer l'effet de l'irritation cutanée sur ces propriétés physiques.
Dans cette étude, nous avons mesuré la vitesse de propagation d'ondes ultrasonores à travers une peau irritée par des tensio-actifs.

\section{Matériel et méthodes}

Vingt-cinq volontaires sains des deux sexes, âgés de 26 à 49 ans, ont participé à létude.

Une solution de tensio-actifs anioniques (ammonium laureth sulphate et ammonium lauryl sulphate) dilués à $10 \%$ a été appliquée sous occlusion sur un avant-bras de chaque volontaire. L'autre avant-bras a servi de témoin non traité. Les tests occlusifs sont restés en contact avec la peau pendant 24 heures, suivies d'une période de repos de 3 heures, puis d'une nouvelle application de tests occlusifs pour une durée de 21 heures.

La mesure du temps de propagation d'ondes ultrasonores (RRTM, resonance running time measurement) a été évaluée en utilisant un Reviscometer ${ }^{\circledR}$ RVM 600 (C + K Electronic, Cologne, Germany) [912]. La sonde de cet appareil est constituée de deux lamelles parallèles distantes l'une de lautre de $2 \mathrm{~mm}$. Lorsqu'on dépose la sonde sur le site à mesurer, une des lamelles de la sonde émet des ondes ultrasonores qui sont alors transmises par la peau et captées par l'autre lamelle à fonction réceptrice. Le temps que prend l'onde pour passer de la lamelle émettrice à la lamelle réceptrice correspond au RRTM exprimé en unités arbitraires. Sur 50 mesures répétitives, le RRTM dans l'air et sur une feuille de polymère de silicone atteignait respectivement $1.724 \pm 21$ et $85 \pm 1$; dès lors la variabilité instrumentale de cet appareil est très basse, de l'ordre de $1.2 \%$.

Les évaluations ont été effectuées à l'initiation de létude ainsi que deux jours après l'enlèvement des tests occlusifs, c'est-à-dire à un moment où le niveau de xérose desquamative atteint son maximum. Le score de rugosité et de desquamation a été déterminé sur une échelle à 5 niveaux (Tableau I).

Les mesures au Reviscometer ${ }^{\circledR}$ ont été réalisées sous température ambiante contrôlée à $21^{\circ} \mathrm{C}$ et à humidité relative de $40 \pm 5 \%$. La pression exercée par la sonde sur la peau était contrôlée. Pour chaque volontaire, 16 mesures pluri-directionnelles de RRTM ont été réalisées sur chaque site, et leur moyenne intraindividuelle a été retenue.

Pour l'ensemble des volontaires, la moyenne interindividuelle des RRTM (M), la déviation standard (DS), la médiane et le coefficient de variation $\left(\mathrm{CV}=10^{2}\right.$ DS. $\left.\mathrm{M}^{-1}\right)$ ont été calculés. Les sites témoins et les sites irrités ont été comparés par le test $\mathrm{t}$ de 
Tableau I Scores cliniques de rugosité et de desquamation. L'attribution de demi-scores permet d'affiner l'appréciation

\section{Grade Etat de la couche cornée}

$\begin{array}{ll}0 & \text { Lisse, pas d'altération } \\ 1 & \text { Xérose légère sous forme de stries blanches ou de quelques squames très fines seulement visible sous la loupe } \\ 2 & \text { Xérose modérée, aspect finement poudreux visible à l'œil nu, ou quelques squames larges, ou surface discrètement rugueuse } \\ 3 & \text { Nettement xérotique, squameux, desquamation sur zone étendue, ou surface nettement rugueuse } \\ 4 & \text { Très xérotique, comme le grade } 3 \text { avec en plus des fissures, des gerçures ou une surface nettement rugueuse }\end{array}$

Student pour échantillons appariés. Une valeur de $\mathrm{p}$ inférieure à 0.05 a été considérée comme statistiquement significative.

\section{Résultats}

Les valeurs moyennes interindividuelles des RRTM étaient similaires sur les deux avant-bras à l'inclusion dans l'étude. Aucune modification significative n'est apparue sur le site témoin à l'issue de létude. En revanche, la moyenne interindividuelle de RRTM sur le site irrité est devenue significativement inférieure $(P<0.0001)$ à celle obtenue pour les sites sains (Fig. 1). La même constatation était apparente pour les médianes qui atteignaient 163 sur le site sain et 99 sur le site irrité. CV était un peu plus faible sur le site sain (32\%) que sur le site irrité (36\%), mais cette différence n'était pas statistiquement significative.

Le score de rugosité et de desquamation était plus sévère sur les sites irrités que sur les sites témoins. Une corrélation linéaire négative $(r=0.71, P<0.01)$ est apparue entre les moyennes intraindividuelles de RRTM et le score clinique de rugosité et de desquamation (Fig. 2).

\section{Discussion}

Lirritation cutanée est un phénomène biologique complexe et fréquemment rencontré. De nombreux xénobiotiques en sont responsables. Les tensio-actifs anioniques en font partie et les conséquences cutanées dépendent de leurs natures moléculaires, de leurs concentrations, de leurs associations, ainsi que de la durée et de la répétition des contacts. Locclusion est également un facteur accroissant l'irritation [15-18].

La cinétique des dégats cutanés faisant suite à des applications occlusives de tensio-actifs est différente selon la cible biologique envisagée. En particulier, les altérations de la structure de la couche cornée deviennent maximales dans les premiers jours suivant l'agression. C'est pourquoi les mesures ont été réalisées après un intervalle libre de deux jours dans cette étude.

Le Reviscometer ${ }^{\circledR}$ a été conçu pour mesurer la vitesse de propagation d'ondes ultrasonores dans la peau [11-13]. Des études ont démontré que la vitesse de propagation de l'onde ultrasonore était proportionnelle à l'augmentation de la rigidité de la peau [8-13].

Figure 1 Moyennes interindividuelles et déviations standard des valeurs de RRTM (unités arbitraires) sur les sites sains et irrités.

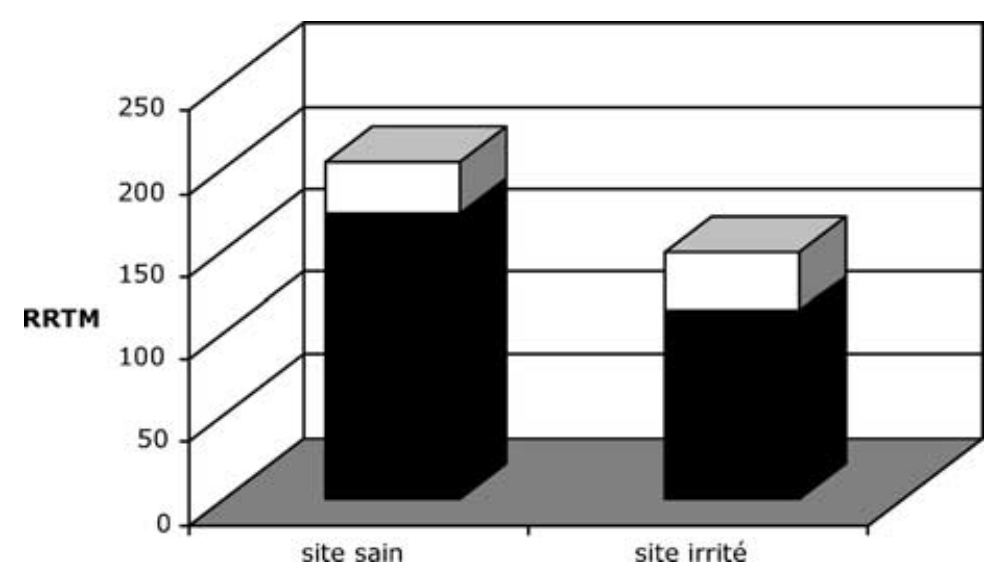




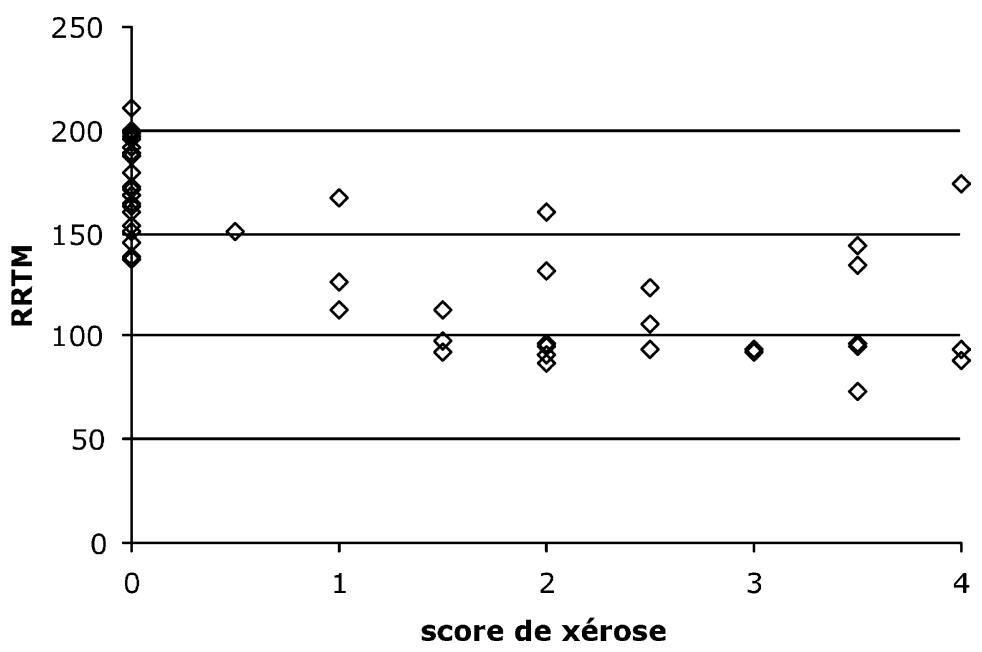

Figure 2 Corrélation linéaire négative $(r=-0.71)$ entre les valeurs moyennes intraindividuelles de RRTM et les scores cliniques de rugosité et de desquamation.
Notre étude révèle qu'un site irrité par une solution de tensio-actifs sous occlusion et qui se présente sous une apparence xérotique, avec une desquamation assez importante, voire une certaine rugosité et modification du relief cutané, a des valeurs de RRTM significativement inférieures à celles obtenues sur la peau intacte. Cette réduction de la valeur RRTM apparaît corrélée à la sévérité des dégâts dans la structure de la couche cornée. Dès lors, la vitesse de propagation de londe ultrasonore augmente sur un site irrité par rapport à un site sain. La variation interindividuelle de la réponse cutanée à des irritants est bien reconnue en pratique clinique. Elle apparaît à la lumière de cette étude pouvoir être objectivée par des mesures de RRTM. Cependant, selon notre expérience, la sensibilité de la mesure du RRTM dans la détection d'une altération de la couche cornée par les tensio-actifs natteint cependant pas celle rapportée pour certaines mesures de propriétés électriques de la couche cornée [19] et de son aspect sous lumière ultraviolette [20, 21].

En conclusion, cette étude démontre que la vitesse de propagation dondes ultrasonores est influencée par létat de surface cutané et qu'elle est nettement accélérée dans la peau très xérotique. Le Reviscometer ${ }^{\mathbb{R}}$ trouve ainsi une nouvelle utilité dans le cadre de mesures quantitatives et non invasives des effets négatifs potentiels de certains produits de soins pour la peau.

\section{References}

1. Oldenhove de Guertechin, L. Surfactant: classification. In: Handbook of detergents Part A: Properties (G. Broze, ed.), pp. 7-46. Marcel Dekker, New York (1999).
2. Polefka, T. Surfactant interactions with skin. In: Handbook of detergents Part A: Properties (G. Broze, ed.), pp. 443-468. Marcel Dekker, New York (1999).

3. Agner,T. and Serup, J. Individual and instrumental variations in irritant patch-test reactions - clinical evaluation and quantification by bioengineering methods. Clin. Exp. Dermatol. 15, 29-33 (1990).

4. Berardesca, E. EEMCO guidance for the assesment of stratum corneum hydratation: electrical methods. Skin Res. Technol. 3, 126-132 (1997).

5. Rogiers, V. and the EEMCO Group. EEMCO guidance for the assesment of the transepidermal water loss in cosmetic sciences. Skin Pharmacol. Appl. Skin Physiol. 14, 117-128 (2001).

6. Fisher,T.W,Wigger-Alberty, W. and Elsner, P. Assesment of 'dry skin': current bioengineering methods and test designs. Skin Pharmacol. Appl. Skin Physiol. 14, 183-195 (2001).

7. Pittet, J.-C. and Beau, P. La mesure biophysique de l'hydratation cutanée in vivo. Ann. Dermatol. Venereol. 129, 123-130 (2002).

8. Pereira, J.M, Mansour, J.M. and Davis, B.R. Analysis of shear wave propagation in skin: application to an experimental procedure. J. Biomech. 23,745-751 (1990).

9. Mridha, M, Odman, S. and Oberg, P.A. Mechanical pulse wave propagation in gel, normal and oedematous tissues. J. Biomech. 25, 1213-1218 (1992).

10. Vexler, A, Polyanski, I. and Gorodetski, R. Evaluation of skin viscoelasticity and anisotropy by measurement of speed of shear wave propagation with viscoelasticity skin analyser. J. Invest. Dermatol. 113, 732-739 (1999).

11. Nizet, J.L, Piérard-Franchimont, C. and Piérard, G.E. Influence of the body posture and gravitational forces on shear wave propagation in the skin. Dermatology 202, 177-180 (2001).

12. Hermanns-Lê, T, Jonlet, F, Scheen, A. and Piérard, G.E. Age and body mass index-related changes in cutaneous shear wave velocity. Exp. Gerontol. 36, 363-372 (2001). 
13. Uhoda, I, Faska, N, Robert, C, Cauwenbergh, G. and Piérard, G.E. Split face study of cutaneous tensile effect of a 2-diemthylaminoethanol (deanol) gel. Skin Res. Technol. 8, 164-167 (2002).

14. Piérard-Franchimont, C, Goffin, V, Uhoda, E. and Piérard, G.E. Ultrasound shear wave propagation in Caucasian hair. Exog. Dermatol. (in press).

15. Van der Valk, P.G.M. and Maibach, H.I. Post-application occlusion substantially increases the irritant response of the skin to repeated short-term sodium lauryl sulfate (SLS) exposure. Contact Dermat. 21, 335-338 (1989).

16. Fluhr, J.W, Lazzerini, S, Distante, F, Gloor, M. and Berardesca, E. Effects of prolonged occlusion on stratum corneum barrier function and water holding capacity. Skin Pharmacol. Appl. Skin Physiol. 12, 193-198 (1999).
17. Warner, R.R, Boissy, Y.L, Lilly, N.A et al. Water disrupts stratum corneum lipid lamellae: damage is similar to surfactants. J. Invest. Dermatol. 113, 960-966 (1999).

18. Hongbo, Z. and Maibach, H.I. Occlusion vs. Skin barrier function. Skin Res. Technol. 8, 1-6 (2002).

19. Goffin, V, Piérard-Franchimont, C. and Piérard, G. Passive sustainable hydration of the stratum corneum following surfactant challenge. Clin. Exp. Dermatol. 24, 308-311 (1999).

20. Piérard-Franchimont, C, Petit, L. and Piérard, G.E. Skin surface patterns of xerotic legs: the flexural and accretive types. Int. Cosmet. Sci. 23,121-126 (2001).

21. Piérard-Franchimont, C. and Piérard, G.E. Beyond a glimpse at seasonal dry skin. Rev. Exog. Dermatol. 1, 3-6 (2002). 